\title{
NOUVELLES CAPTURES DE NYCTERIBIIDAE (DIPTERA, PUPIPARA) EN FRANCE
}

\author{
Par J.-C. BEAUCOURNU
}

Nous avons récolté ou reçu pour identification un certain nombre de Nycteribies qui n'entraient pas, par leur origine géographique, dans le cadre d'une précédente note sur les « Ectoparasites des Chiroptères de l'Ouest de la France ». Nous groupons ici ces quelques captures (1).

\section{Genre Penicillidia Kolenati 1882.}

\section{- P. CONSPICUA Speiser 1901.}

Ce parasite est essentiellement inféodé à Miniopterus schreibersi (Kuhl) et sa répartition en France doit se superposer intégralement à celle de son hôte.

Il est signalé des départements suivants :

Ain (Aellen, 1955) ; Charente (Beaucournu, 1961) ; Drôme (Falcoz, 1923) ; Pyrénées-Orientales (Leclercq et Théodoridès, 1950).

\section{Nouvelle capture :}

Ariège, Massat, Grotte du Queire (18-7-61) : 1 ơ sur Miniopterus schreibersi.

- P. DUFOURI (Westwood, 1835).

Cette Nyctéribie, qui peut se rencontrer sur le Minioptère, parasite particulièrement Myotis myotis (Bechst.) et Myotis blythi oxygnathus (Montic.).

Elle est plus commune en France que la précédente et est signalée des départements suivants: Ain (Aellen), Ardèche (Falcoz), Ariège (Aellen ; Falcoz). Aude, Basses-Pyrénées (Falcoz), Côte-d’Or (Larrousse, 1924), Gard, HauteGaronne (Falcoz), Haute-Savoie, Jura (Aellen), Maine-et-Loire, Mayenne (Beaucournu).

\section{Nouvelles captures :}

Ardèche, Châteaubourg, Grotte des Ours (24-6-50): 1 ơ, 2 \%, sur Myotis myotis (1 $q$ parasitée par un Laboulbeniacé) (Balazuc, coll.).

(1) Les captures citées sans mention de collecteur ont été opérées par nousmême. Les autres sont le fait de MM. Balazuc, Salvayre, Lucas et Colin, que nous remercions vivement. 
Même endroit (5-5-52): 1 ơ, 3 \&, sur M. myotis ou Miniopterus schreibersi (Balazuc, coll.).

Ardèche, Gras, Grotte de Fabrège (8-8-51): $2 \delta^{\prime}, 2$, sur M. myotis $(1 q$ parasitée par un Laboulbeniacé) (Balazuc, coll.).

Ardèche, Ruoms, Grotte de Baume-Grenas (Biosp. 443) (24-8-58): 1 б, 1 , sur $M$. myotis (Balazuc, coll.) (1).

Même endroit (29-8-58): 1 đ', 2 \%, sur M. myotis (Balazuc, coll.).

Ariège, Massat, Grotte du Queire (18-7-61): 11 б', 17 \%, sur M. myotis; 5 đै, 4 \&, sur Miniopterus schreibersi.

\section{Genre Basilia Mir. Ribeiro 1903.}

- B. NATTERERI (Kolenati, 1857).

Nous ne possédons que fort peu de renseignements sur cette espèce. Elle a été signalée du Maine-et-Loire (Théodor et Moscona, 1954), de Loire-Atlantique et de Mayenne (Beaucournu).

Nouvelles captures :

Ariège, Massat, Grotte du Queire (17-12-61): 2 \%, sur Myotis nattereri (Kuhl).

Lot-et-Garonne, Xaintrailles, La Glacière (13-2-61): 2 ơ, 3 q, sur Myotis nattereri. Cohabitation avec B. nana.

Même endroit (16-12-61): $1 \delta^{*}, 1$, sur Myotis nattereri.

- B. NANA Théodor 1954.

Cette petite Nyctéribie, longtemps confondue avec $B$. nattereri, est également de répartition incertaine. Elle est connue des départements suivants : Loire-Atlantique, Mayenne, Morbihan, Sarthe (Beaucournu), Seine-Maritime (Théodor et Moscona), Vendée (Beaucournu).

Nouvelles captures:

Lot-et-Garonne, Xaintrailles, La Glacière (13-2-61) : 1 q, sur Myotis nattereri.

Même endroit (16-12-61): 1 ๙, sur Myotis bechsteini (Kuhl).

Même endroit (6-1-62): 1 q, sur Myotis bechsteini.

\section{Genre Nycteribia Latreille 1796.}

\section{- N. (NYCTERIBIA) PEDICULARIA Latreille 1805.}

Il existe un certain nombre de captures rapportées à cette espèce, pour la France. Malheureusement, ces mentions sont antérieures, pour la grande majo-

(1) Captures effectuées sur chauves-souris en essaim : \&...Ces chauves-souris étaient grouillantes de parasites (Nyctéribies, Acariens du genre Spinturnix)... (Balazuc, in Heerdt, Sluiter et Balazuc, 1959). 
rité, aux travaux de Théodor (1954) et Théodor et Moscona (1954) qui ont scindé l'espèce type en trois bonnes espèces. Il semble que l'on doive rapporter la plupart des Nyctéribies signalées à $N$. (N.) latreillii (Leach). En effet, $N$. (N.) pedicularia parasite surtout Myotis capaccinii (Bonap.), Chiroptère peu commun ; N. (N.) latreillii : M. myotis et $M$. blythi oxygnathus; $N$. (N.) kolenatii Théodor : $M$. daubentoni (Kuhl). C'est ainsi que le spécimen signalé par Falcoz (1923) sur $M$. oxygnathus ( $=M$. blythi oxygnathus), de Bugarach (Aude), se rapporte vraisemblablement à $N$. latreillii. Par contre, il n'est guère possible de savoir à quoi correspondent les captures de Larrousse (1924) sur Rhinolophus hipposideros, ou de Balazuc, Dresco, Henrot et Nègre (1951) sur Myotis emarginatus, hôtes très exceptionnels pour un Insecte ectoparasite (Beaucournu, 1961). Signalons qu'il y a au moins une capture certaine de N. pedicularia, en France, sur Myotis capaccinii, d'ailleurs, en Ariège (Aellen). Théodor et Moscona (1954) la citent également de notre nays, mais sans référence précise.

\section{Nouvelles captures :}

Pyrénées-Orientales, Salses, souterrains du château (?-12-61) : 1 \%, sur Myotis capaccinii (1) (Salvayre, coll.).

Ardèche, Ruoms, Grotte de Baume-Grenas (Biosp. 443) (29-8-58) : 1 ઠే, 2 q, sur Myotis capaccinii (Balazuc, coll.).

-N. (NYCTERIBIA) LATREILLII (Leach, 1817).

Cette espèce est certainement très rare dans l'Ouest. Elle semble, par contre, commune dans la moitié Sud de la France. Elle est jusqu'à maintenant signalée d'Ariège, du Jura (Aellen) et du Morbihan (Beaucournu).

\section{Nouvelles captures :}

Ardèche, Gras, Grotte de Fabrège (8-8-51) : 1 \%, sur Myotis myotis (Balazuc, coll.).

Ardèche, Châteaubourg, Grotte des Ours (24-6-50): 1 , sur M. myotis (Balazuc, coll.).

Même endroit (5-5-52): 1 \&, sur $M$. myotis (Balazuc, coll.).

Ariège, Massat, Grotte du Queire (18-7-61): $5 \delta^{*}, 2 q$, sur M. myotis; 1 ơ, sur $M$. blythi oxygnathus.

\section{- N. (NYCTERIBIA) SCHMIDLI Schiner 1853.}

Cette Nyctéribie est presque exclusivement inféodée à Miniopterus schreibersi et on peut faire à son propos les mêmes remarques qu'au sujet de Penicillidia conspicua.

(1) Un seul hôte a été étudié ne livrant que cette Nyctéribie et des Acariens (Salvayre, in litt., 18-3-62). 
Elle est connue de l'Ain (Aellen), Ariège (Aellen, Falcoz), Charente (Beaucournu), Doubs (Aellen), Drôme, Haute-Garonne, Hérault (Falcoz), PyrénéesOrientales (Leclercq et Théodoridès), Tarn (Falcoz).

\section{Nouvelles captures :}

Ariège, Massat, Grotte du Queire (18-7-61): 2 , sur $M$. myotis ; 1 , sur M. blythi oxygnathus; 11 ơ, 12 \&, sur Miniopterus schreibersi.

Hautes-Pyrénées, Frechet-Aure, Grotte du village (?-4-62): 1 \&, sur Miniopterus schreibersi (spécimen parasité par un Laboulbeniacé) (Lucas, coll.).

\section{- N. (ACHROCHOLIDIA) VEXATA Westwood 1835.}

Parasite du Myotis myotis et du M. blythi oxygnathus, assez rare partout, semble-t-il.

Elle est connue de l'Ariège (Aellen), de l'Oise (Balazuc, Dresco, Henrot et Nègre), des Pyrénées-Orientales (Leclercq et Théodoridès), de la Sarthe (Beaucournu) et de la Seine (Balazuc et coll.).

\section{Nouvelles captures :}

Ardèche, Châteaubourg, Grotte des Ours (24-6-50) : 1 đै, 1 \%, sur M. myotis (Balazuc, coll.).

Même endroit (5-5-52): 1 \%, sur M. myotis (Balazuc, coll.).

Ardèche, Châteaubourg, Grotte des Ours (5-5-52): 1 , sur $M$. myotis ou Miniopterus schreibersi (Balazuc, coll.).

Ariège, Massat, Grotte du Queire (18-7-61): 4 o', 6 \%, sur M. myotis ; 1 ơ, sur $M$. blythi oxygnathus.

Lot-et-Garonne, Xaintrailles, La Glacière (13-2-61) : 1 q, sur M. myotis.

\section{- N. (STYLIDIA) BIARTICULATA Hermann 1804.}

Cette espèce, parasite des Rhinolophidés, est la seule, à notre connaissance, à présenter une densité appréciable sur l'ensemble de la France. La liste des départements donnés ci-dessous est certainement incomplète :

Ain (Aellen), Alpes-Maritimes (Falcoz), Ardèche (Aellen ; Leclercq et Théodoridès ; Falcoz), Ariège (Aellen), Aude (Falcoz), Bouches-du-Rhône, Doubs (Aellen), Gard (Falcoz; Leclercq et Théodoridès), Hautes-Alpes (Falcoz), Haute-Savoie (Aellen), Hérault (Falcoz), Loire-Atlantique (Beaucournu), Lot (Falcoz), Maine-et-Loire, Mayenne, Morbihan, Sarthe (Beaucournu).

\section{Nouvelles captures:}

Jura, Saint-Claude, Grotte inférieure de la Grusse (?-12-56) : 1 ơ, sur parois (Colin, coll. ; Dresco, leg.).

Pyrénées-Orientales, Fuilla, Grotte de Fuilla (?-12-61) : 1 , sur Rhinolophus euryale Blasius (Salvayre, coll.).

Pyrénées-Orientales, Banyuls, Grotte de Pouade (21-1-62) : 3 б, sur R. ferrum-equinum (Schreber) ; 8 s, 1 \%, sur $R$. euryale. 


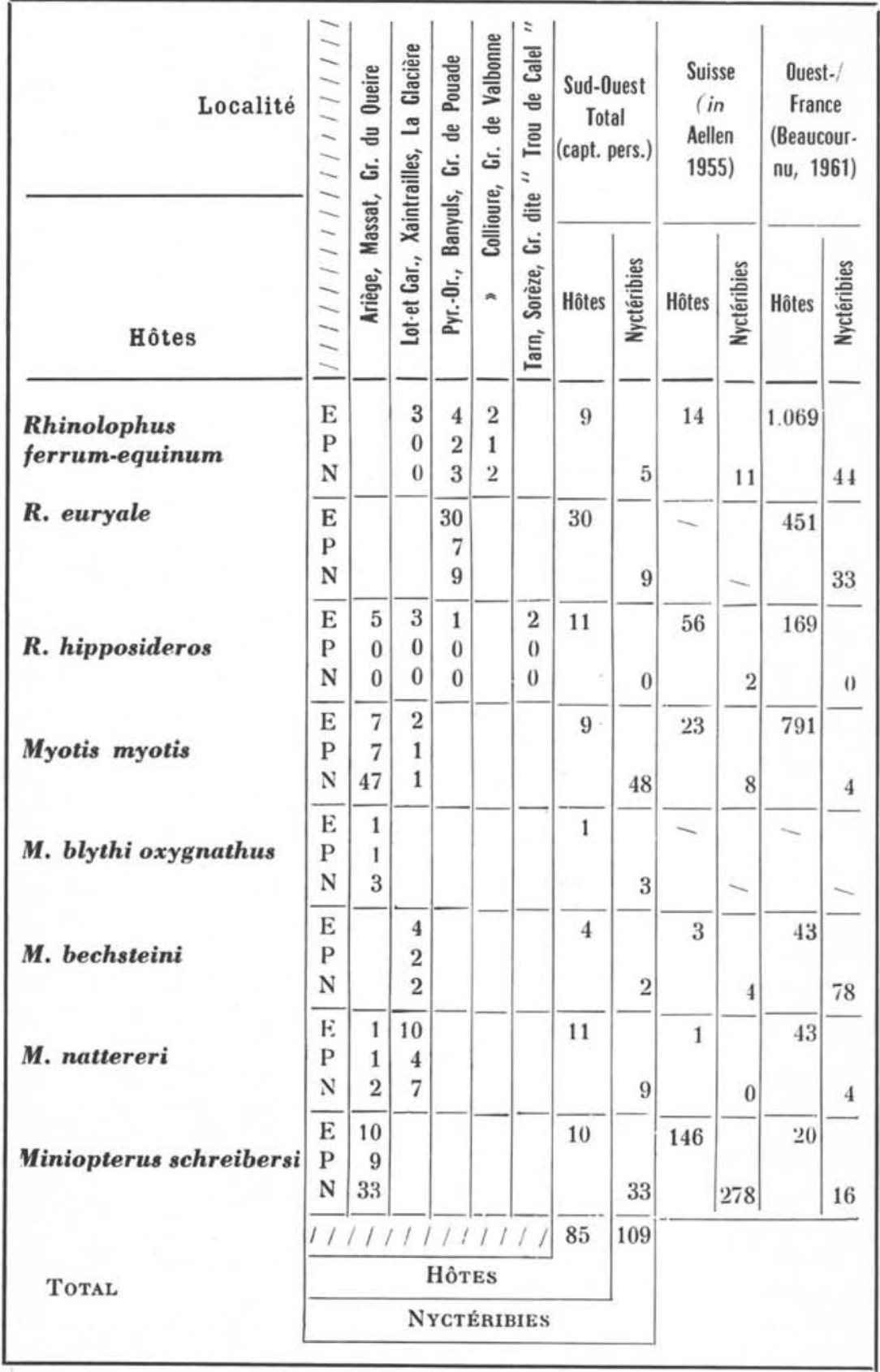

E : Nombre de chauves-souris étudiẻes.

P. : Nounbre de chauves-souris parasitées par des nyctèribies.

N. : Nombre de nyctéribies récoltées. 
Pyrénées-Orientales, Collioure, Grotte de Valbonne (31-7-61): nombreuses pupes sur parois $\left(\widehat{\delta}^{\circ}\right.$, ,,+$)$.

Même endroit (21-1-62): 2 , sur $R$. ferrum-equinum.

\section{Remarques}

Il ne nous est pas possible de connaître avec précision le nombre de Chiroptères sur lesquels ont été prélevés les Pupipares qui nous ont été adressés par nos correspondants. Par contre, il nous est facile, pour ceux que nous avons personnellement examinés, d'établir un parallèle entre les résultats que nous avons précédemment obtenus dans l'Ouest de la France et ceux que nous consignons ici. Il va sans dire que la même méthode de prélèvement des parasites a été utilisée, les hôtes étant isolés dès la capture et relâchés après examen.

Le tableau ci-joint donne dans ses deux dernières colonnes, pour chacune des espèces de chauve-souris que nous avons rencontrées dans le Sud-Ouest, non seulement nos résultats pour l'Ouest, mais également, à titre de comparaison, ceux extraits d'Aellen (1955) concernant la Suisse.

Rhinolophus ferrum-equinum: Les individus examinés dans les Pyrénées-Orientales et le Lot-et-Garonne étaient isolés, en sommeil hivernal. Le pourcentage de Nyctéribies par hôte est de 0,55. Aellen obtient un chiffre de même ordre pour la Suisse avec 0,8. Dans l'Ouest, nous tombons à 0,04!

Rhinolophus euryale: En l'absence de captures suisses, nous ne pouvons que comparer nos résultats personnels. A la Grotte de Pouade (PyrénéesOrientales), sur des Euryale en colonie hivernale compacte : 0,3. Dans l'Ouest : 0,075 pour l'ensemble. Ce taux remonte toutefois à 0,1 si l'on ne considère que les hôtes étudiés dans les mêmes conditions de saison et de groupement qu'à Pouade.

Rhinolophus hipposideros est, dans l'ensemble, égal à lui-même. Les deux Nyctéribies d'Aellen sur 56 hôtes ne sont pas près d'être égalées : 0 sur 11 dans le Sud-Ouest, 0 sur 169 dans l'Ouest.

Myotis myotis : Nous obtenons dans le Sud-Ouest un taux extraordinairement fort avec 5,33 par hôte. Il est vrai que, sur les 9 étudiés, 7 provenaient d'un essaim estival où les conditions sont éminemment favorables à la pullulation des Nyctéribies. Pour la Suisse, le chiffre est de 0,35. Dans l'Ouest, nous atteignons peut-être un record de rareté : 0,005 pupipare par chauve-souris, et cela, rappelons-le, sur près de 800 grands murins.

Le cas de Myotis blythi oxygnathus est sans intérêt, car isolé. Il est vraisemblable que son taux d'infestation doit être très voisin de celui de $M$. myotis.

De même, les chiffres que nous pouvons obtenir à propos de Myotis Ans. de Parasitologie, T. XXXVII, N $3 .-1962$. 
bechsteini sont sujets à réserves pour le Sud-Ouest comme pour la Suisse, car basés sur trop peu de spécimens. Dans l'ensemble, il s'avère cependant que cette chauve-souris compte parmi les plus riches en Nyctéribies (sinon en espèces, du moins en spécimens).

Myotis nattereri est assez fréquemment parasité dans nos deux séries de captures : dans l'Ouest, il s'agit essentiellement de Siphonaptères ; dans le SudOuest, de Nyctéribies (1).

Miniopterus schreibersi, enfin, se révèle riche en parasites (Nyctéribies presque uniquement), en quelque localité qu'on l'étudie. A Massat (Ariège), nous obtenons un taux de 3,3 , et encore faut-il souligner que l'un des dix hôtes étudiés le fut en hiver, isolé, et ne livra aucun ectoparasite. Toutefois, les conditions de captures des neuf autres, en essaim estival, doivent s'accompagner des mêmes réserves qu'à l'égard de $M$. myotis. En Suisse, le taux moyen s'élève à 1,8 , ce qui est encore considérable. En Charente, enfin, nous obtenons un taux de 0,8 sur 20 spécimens en essaim d'hivernage. Nous tenons toutefois à préciser que ce taux est sujet à caution, car ces hôtes furent assez rapidement étudiés et dans de mauvaises conditions.

Nous espérons avoir par cette courte note quelque peu contribué à la connaissance de la répartition des Nyctéribies françaises sans doute, mais aussi à l'étude de l'équilibre hôte-parasite dont les modalités en fonction de la latitude sont encore très mal connues.

\section{BIBLIOGRAPHIE}

Aellen (V.), 1955. - Etude d'une collection de Nycteribiidae et de Streblidae (Diptera, Pupipara) de la région paléarctique occidentale, particulièrement de la Suisse. Bull. Soc. Neuchât. Sc. Nat., $78,81$.

Balazuc (J.), Dresco (E.), Henrot (H.) et Nègre (J.), 1951. - Biologie des carrières souterraines de la région parisienne. Vie et milieu, 2, 301.

Balcells (E. R.), 1956. - Estudio biologico y biometrico de Myotis nattereri (Chir. Vespertilionidae). Publ. Inst. Biol. Aplic., 23, 37.

Beaucournu (J. C.), 1961. - Ectoparasites des chiroptères de l'Ouest de la France, I. Bull. Soc. Scient. Bretagne (sous presse).

FALCoz (L.), 1923. — Biospeologica, XLIX, Pupipara (Diptères) (1 ${ }^{\text {re }}$ série). Arch. Zool. Exp. Gen., 61, 521.

(1) Balcells (1956), étudiant cette chauve-souris aux environs de Barcelone, donne les chiffres suivants : « Diptères Pupipares, Penicillidia dufouri: 5 o et 3 ơ réparties sur 80 exemplaires adultes. Deux ou trois exemplaires de Basilia nana par hôte. Aphaniptères (= Siphonaptères) : 5 ot et 2 O de Ischnopsyllus simplex sur les mâles de $M$. nattereri. \$ (ceux-ci étant au nombre de 12 d'après des renseignements précédemment donnés dans le texte). 
Heerdt (P. F. van), Sluiter (J. W.) et Balazuc (J.), 1959. - Suite des recher. ches sur les Chiroptères dans les grottes de l'Ardèche. Campagnes de 1957-1958. Bull. Soc. Linn. Lyon, 28, 165.

Leclerce (M.) et ThÉodoridès (J.), 1950. - Some ectoparasites of birds and Mammals observed recently in France. Ent. Month. Mag., 86, 74.

Neveu-Lemaire (M.), Joyeux (C.), Larrousse (F.), Isobe (M.) et Lavier (G.), 1924. - Parasites de chauves-souris de la Côte-d'Or. C.R. Cong. Soc. Sav., 274.

Tнв́onon (O.), 1954. - Nycteribiidae, in E. Lindner, Flieg. Palearlit. Reg., 66a. - et Moscosa (A.), 1954. - On bats parasites in Palestine. I : Nycteribiidae, Streblidae, Hemiptera, Siphonaptera, Parasitology, 4h, 157.

[Laboratoire de Parasitologie et Zoologie appliquée ( $\mathrm{P}^{\mathrm{r}}$ DoBY) de la Faculté de Médecine et de Pharmacie de Rennes] 